\title{
Platelet Transfusion Improves Liver Function in Patients with Chronic Liver Disease and Cirrhosis
}

\author{
Takehito Maruyama, ${ }^{1}$ Soichiro Murata, ${ }^{2}$ Kazuhiro Takahashi, ${ }^{1}$ Takafumi Tamura, ${ }^{1}$ \\ Reiji Nozaki, ${ }^{1}$ Naoya Ikeda, ${ }^{1}$ Kiyoshi Fukunaga, ${ }^{1}$ Tatsuya Oda, ${ }^{1}$ Ryoko Sasaki ${ }^{1}$ and \\ Nobuhiro Ohkohchi ${ }^{1}$ \\ ${ }^{1}$ Department of Surgery, Division of Gastroenterological and Hepatobiliary Surgery, and Organ Transplantation, \\ University of Tsukuba, Tsukuba, Ibaraki, Japan \\ ${ }^{2}$ Department of Surgery, Teikyo University Chiba Medical Center, Ichihara, Chiba, Japan
}

\begin{abstract}
Chronic liver disease (CLD), such as hepatitis $C$, is a progressive disease consisting of the destruction and regeneration of the liver parenchyma, leading to fibrosis and cirrhosis. Platelets contain various growth factors and may play important roles in liver regeneration. Thus, to investigate whether platelet transfusion improves liver function in patients with CLD and cirrhosis, we conducted an exploratory clinical trial. The study included 10 patients with CLD and cirrhosis (Child-Pugh class A or B), who all presented thrombocytopenia (platelet counts between 50,000 and 100,000/ $/ \mu \mathrm{l}$ ). The subjects received 10 units of platelet concentrate once a week for 12 weeks. They were followed up for 9 months after the last transfusion. One patient discontinued platelet transfusion because of pruritus, and 2 patients discontinued because of platelet transfusion refractoriness. One patient was excluded from the analysis for receiving a procedural treatment after 12 platelet transfusions. Thus, the remaining 6 patients were analyzed. The platelet count did not increase significantly after the last transfusion. Significant improvement of serum albumin was observed at 1 month and 3 months after the last transfusion. Serum cholinesterase improved significantly at 1 week, 3 months, and 9 months after the last transfusion. Serum hyaluronic acid showed a tendency toward improvement after the last transfusion. In conclusion, platelet transfusion improved some of the indicators of liver function in patients with CLD and cirrhosis, though adverse events related to platelet transfusion were observed in some patients. Platelet increment therapy could be a new strategy for treating CLD and cirrhosis.
\end{abstract}

Keywords: chronic liver disease; liver cirrhosis; liver function; platelet transfusion; platelet transfusion refractoriness

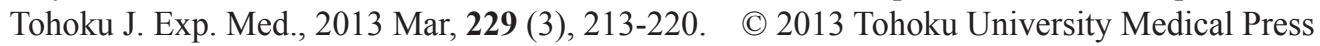

\section{Introduction}

Chronic liver disease (CLD) is a progressive disease consisting of the destruction and regeneration of the liver parenchyma leading to fibrosis and cirrhosis. Liver cirrhosis is the end stage of CLD and a major life-threatening health problem worldwide. One of common complications in patients with CLD and cirrhosis is thrombocytopenia (Afdhal et al. 2008). Thrombocytopenia occurs in up to $64 \%$ of cirrhotic patients (Bashour et al. 2000). Patients with advanced end-stage liver disease tend to have a higher degree of thrombocytopenia than those patients with compensated cirrhosis (Adinolfi et al. 2001; Giannini et al. 2002). Patients with thrombocytopenia are sometimes unable to receive antiviral therapy with a sufficiently high dose of interferon (IFN) to treat hepatitis virus infection or are unable to undergo curative surgery for hepatocellular carcinoma (HCC). In addition to thrombocytopenia, patients with CLD and cirrhosis experience serious complications, such as ascites, spontaneous bacterial peritonitis, hepatic encephalopathy, portal hypertension, esophagogastric variceal hemorrhage, and hepatorenal syndrome (D'Amico et al. 2006; Heidelbaugh and Sherbondy 2006). When medications or procedural treatments fail to control various symptoms related to CLD and cirrhosis, the only effective therapy is liver transplantation (Merion et al. 2005). However, liver transplantation is associated with serious problems, such as donor shortage, surgical complications, organ rejection, and high cost (Neuberger and James 1999; Taylor et al. 2002; Mueller et al. 2004; Porrett et al. 2009). Due to the shortage of donor organs, patients needing liver transplantation often wait for years, and some of them die while waiting for transplantation. Therefore, alternative therapies are necessary to treat patients with

Received December 18, 2012; accepted February 5, 2013. Published online March 2, 2013; doi: 10.1620/tjem.229.213.

Correspondence: Nobuhiro Ohkohchi, M.D., Ph.D., Department of Surgery, Division of Gastroenterological and Hepatobiliary Surgery, and Organ Transplantation, University of Tsukuba, 1-1-1 Tennodai, Tsukuba 305-8575, Japan.

e-mail: nokochi3@md.tsukuba.ac.jp 
CLD and cirrhosis.

Platelets play an important role not only in hemostasis but also in wound healing and tissue regeneration (Anitua et al. 2004; Diegelmann and Evans 2004). They contain various growth factors, such as platelet-derived growth factor (PDGF), hepatocyte growth factor (HGF), epidermal growth factor (EGF), insulin-like growth factor-1 (IGF-1), and transforming growth factor-beta (TGF- $\beta$ ) (Assoian et al. 1983; Oka and Orth 1983; Nakamura et al. 1986; Miyazono and Takaku 1989), which are required for tissue regeneration. Platelets induce hepatocyte proliferation by releasing HGF and IGF-1 (Matsuo et al. 2008). In addition, liver sinusoidal endothelial cells (LSECs), which constitute the sinusoidal wall, are involved in liver regeneration (Malik et al. 2002), and platelets stimulate LSECs to promote hepatocyte proliferation (Kawasaki et al. 2010). Thrombocytosis promotes liver regeneration not only in normal livers but also in fibrotic livers (Murata et al. 2007; Murata et al. 2008b; Myronovych et al. 2008; Watanabe et al. 2009). Hepatic stellate cells (HSCs) play a major role in liver fibrosis (Friedman 2008). Platelets suppress the activation of HSCs by releasing adenosine 5'-triphosphate (Ikeda et al. 2011), and thrombocytosis reduces liver fibrosis (Murata et al. 2008b; Watanabe et al. 2009). Taken together, these findings indicate that platelets promote liver regeneration and reduce liver fibrosis and that platelet increment therapy may be effective for patients with CLD and cirrhosis. There have been no reports on platelet transfusion for patients with CLD and cirrhosis to improve liver function. The purpose of this exploratory clinical trial is to investigate the efficacy, safety, and feasibility of platelet transfusion in patients with CLD and cirrhosis.

\section{Materials and Methods}

\section{Patients}

This exploratory clinical trial was approved by the institutional review board of Tsukuba University Hospital and was performed in accordance with the Declaration of Helsinki. This trial was registered in the UMIN Clinical Trials Registry system (http://www.umin.ac.jp/. NO. UMIN000001164).

The proposal was designed to include 10 patients with CLD and cirrhosis. Inclusion criteria were as follows: patients aged over 20 years; Child-Pugh class A or B; a platelet count between 50,000 and
$100,000 / \mu 1$ in the previous year; no malignancy, including viable HCC; performance status 0 or 1 ; no serious cardiovascular or cerebrovascular disease; life expectancy of at least 12 months; and ability to provide informed consent. Exclusion criteria were as follows: current viable HCC; pregnancy, lactation, or the desire to get pregnant in women; history of shock on platelet transfusion; bone marrow transplantation recipient (except auto-transplantation); immunosuppressive patients; IgA deficiency or other plasma protein deficiency; and inability to provide informed consent. All patients signed the written informed consent.

\section{Pre-treatment Assessment}

Prospective patients visited the Department of Surgery at Tsukuba University Hospital. Physical examinations were performed, and their intravenous blood was collected to assess liver function, liver fibrosis markers, complete blood count, coagulation profile, urea and electrolyte status, and human immunodeficiency virus serology. The Child-Pugh class and score, i.e., serum albumin, total bilirubin (T-Bil), prothrombin time activity, ascites, and encephalopathy, were used to evaluate the overall condition of the patients. Abdominal ultrasonography with Doppler assessment and computed tomography (CT) scan were performed to assess ascites and disease in the liver and portal system.

\section{Platelet Transfusion}

Irradiated leukocyte-reduced platelet concentrate (Japanese Red Cross Society, Tokyo, Japan) was used as platelet transfusion. Ten units of platelet concentrate contained more than $2.0 \times 10^{11}$ platelets. Each patient received 10 units of platelet concentrate once a week for 12 weeks via the peripheral vein. To assess whether the patients were refractory to platelet transfusion, we obtained their blood before transfusion and at 1 hour after transfusion each time. The 1-hour corrected count increment was calculated as the difference between the platelet count at 1 hour after transfusion and the platelet count before transfusion, multiplied by the body surface area $\left(\mathrm{m}^{2}\right)$ and divided by the number of platelets transfused times $10^{-11}$ (Daly et al. 1980). Platelet transfusion refractoriness (PTR) was defined as a 1-hour corrected count increment of less than $5,000 / \mu 1$ on 2 sequential occasions (Daly et al. 1980). When PTR was diagnosed, anti-human leucocyte antigen (HLA) and anti-human platelet antigen (HPA) antibodies were investigated. If either anti-HLA or anti-HPA antibodies were identified, patients discontinued platelet transfusion.

\section{Follow-up}

The protocol for this trial is shown in Fig. 1. During platelet

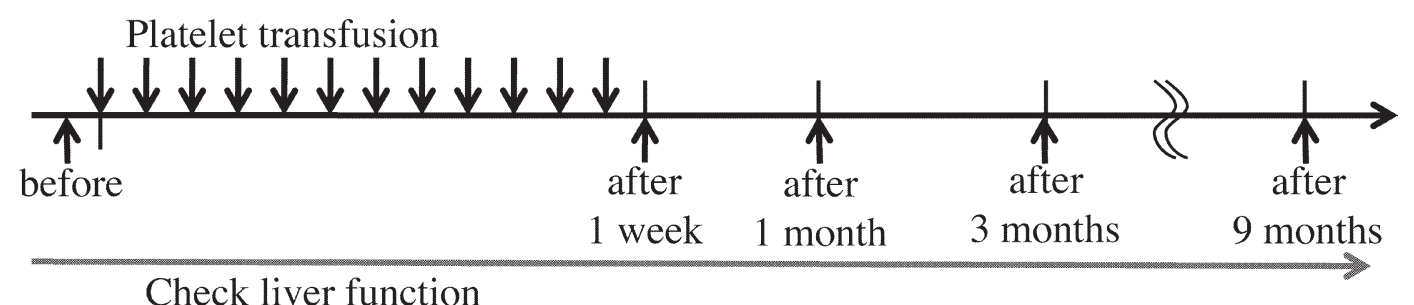

Fig. 1. Trial protocol.

Patients received 10 units of platelet concentrate once a week for 12 weeks. Liver function was monitored by blood examination before the trial, every week during platelet transfusion, and at 1 week, 1 month, 3 months, and 9 months after the last transfusion. Abdominal ultrasonography and computed tomography scan were examined before the trial and at 1,3 , and 9 months after the last transfusion. 
transfusion, patients were followed weekly by physical examination, including liver function, liver fibrosis markers, complete blood count, coagulation profile, and urea and electrolyte status. And then, patients were followed at 1 week and 1 month, 3 months, and 9 months after the last transfusion. Specifically, the concentrations of serum albumin, cholinesterase, aspartate aminotransferase (AST), alanine aminotransferase (ALT), T-Bil, and prothrombin time activity were monitored as markers of liver function, and the concentrations of hyaluronic acid, type IV collagen, and procollagen III peptide (P-III-P) were monitored as markers of liver fibrosis. We examined their livers by abdominal ultrasonography and CT scan to evaluate liver tumors and ascites at 1, 3, and 9 months after the last transfusion. As a marker of regeneration, liver volume was assessed by CT volumetry before and after platelet transfusion. Patients did not receive antiviral therapies, such as IFN, nucleotide analogue, or protease inhibitor, starting 4 weeks before the start of the trial through the end of trial. Medication was unchanged before and after platelet transfusion. Patients who changed medications and received procedural treatments were excluded from this analysis. The primary end point of this trial was to assess the efficacy of platelet transfusion on liver function.

\section{Statistical Analysis}

The changes in data from the values before platelet transfusion to 1 week and 1 month, 3 months, and 9 months after the last transfusion were analyzed. All data are shown as the means \pm standard deviations. Statistical analyses were performed with 1-way repeated measures analysis of variance with Dunnett's test. In all cases, $p$ values $<0.05$ were considered significant.

\section{Results}

\section{Subjects}

Between September 2008 and June 2010, 10 patients with CLD and cirrhosis were recruited into this trial (Table 1). Seven patients were males, and 3 patients were females. The mean age of this trial population was 67.0 years (range: 58 to 72 years). Eight patients had hepatitis C, 1 had hepatitis $\mathrm{B}$, and 1 suffered from non-alcoholic steatohepatitis (NASH). Six patients were classified as Child-Pugh class
A, and the remaining 4 patients were Child-Pugh class B. Although 6 patients had received IFN therapy, none had achieved sustained virological response. Two patients had a history of HCC. They were treated with radiofrequency ablation (RFA), and none had a viable HCC at the start of this trial.

\section{Follow-up and Adverse Events}

Patient 1 chose to discontinue platelet transfusion because of pruritus after the first transfusion. Patient 4 developed mild facial pruritus during the second transfusion. The patient was given injections of hydrocortisone prior to platelet transfusion to prevent side effects, and he continued platelet transfusion without pruritus. Patient 5 developed mild hives during the first transfusion. The patient continued platelet transfusion without hives by receiving injections of hydrocortisone and anti-histamine agent before platelet transfusion. Patient 6 discontinued platelet transfusion after the third transfusion because she was diagnosed as PTR and was positive for anti-HPA antibody. Patient 8 was refractory to platelet transfusion at the first transfusion. She discontinued platelet transfusion after the second transfusion because she was positive for antiHLA antibody. Although patient 9 received platelet transfusion 12 times, he was excluded from the analysis because he received a balloon-occluded retrograde transvenous obliteration (B-RTO) for the treatment of a large splenorenal shunt at 4 months after the last transfusion. Thus, a total of 6 patients were followed up until the end of this trial (Fig. 2). No patients exhibited signs of deteriorated liver function, such as Child-Pugh score, serum AST, ALT, T-Bil, and prothrombin time activity (data not shown). Abdominal ultrasonography and CT scan revealed no new liver tumors and no appearance of ascites in any patients after platelet transfusion.

\section{Changes in Data after Platelet Transfusion}

Changes in the mean platelet count of the analyzed

Table 1. Patient characteristics.

\begin{tabular}{lcccccccl}
\hline & Age & Gender & Diagnosis & $\begin{array}{c}\text { Child-Pugh } \\
\text { class (score) }\end{array}$ & $\begin{array}{c}\text { IFN } \\
\text { history }\end{array}$ & $\begin{array}{c}\text { Transfusion } \\
\text { history }\end{array}$ & $\begin{array}{c}\text { Pregnancy } \\
\text { history }\end{array}$ & \multicolumn{1}{c}{ Comorbidity } \\
\hline Patient 1 & 64 & Male & HBV, LC & B (7) & - & - & - & HCC, Post-RFA \\
Patient 2 & 58 & Male & NASH, LC & A (6) & - & - & - & DM \\
Patient 3 & 71 & Male & HCV, LC & A (6) & - & + & - & Esophageal cancer, Post-CRT \\
Patient 4 & 67 & Male & HCV, LC & B (7) & - & - & - & HCC, Post-RFA, DM \\
Patient 5 & 71 & Male & HCV, LC & B (7) & + & + & - & \\
Patient 6 & 72 & Female & HCV, LC & A (5) & + & + & + & \\
Patient 7 & 68 & Female & HCV, LC & A (5) & + & + & + & Post-gastrectomy, HT \\
Patient 8 & 70 & Female & HCV, LC & A (5) & + & + & + & \\
Patient 9 & 59 & Male & HCV, LC & B (8) & + & + & - & \\
Patient 10 & 68 & Male & HCV, LC & A (6) & + & + & - & DM \\
\hline
\end{tabular}

CRT, chemoradiotherapy; DM, diabetes mellitus; HBV, hepatitis B virus; HCC, hepatocellular carcinoma; HCV, hepatitis C virus; HT, hypertension; IFN, interferon; LC, liver cirrhosis; NASH, non-alcoholic steatohepatitis; RFA, radiofrequency ablation. 


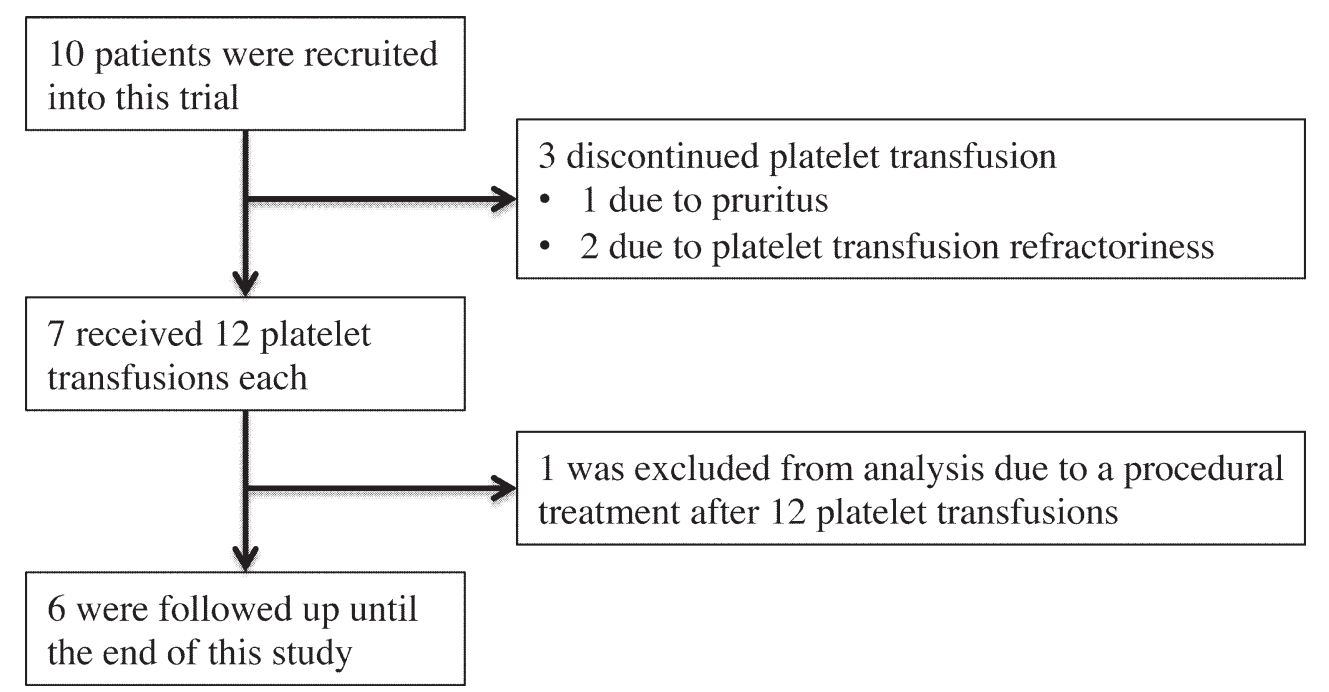

Fig. 2. Follow-up of patients.

Ten patients were recruited into this trial. One patient chose to discontinue platelet transfusion due to pruritus, and 2 patients discontinued due to platelet transfusion refractoriness. Seven patients received 12 platelet transfusions each. One patient was excluded from the analysis because he received a procedural treatment after 12 platelet transfusions. Thus, 6 patients were followed up until the end of this trial.

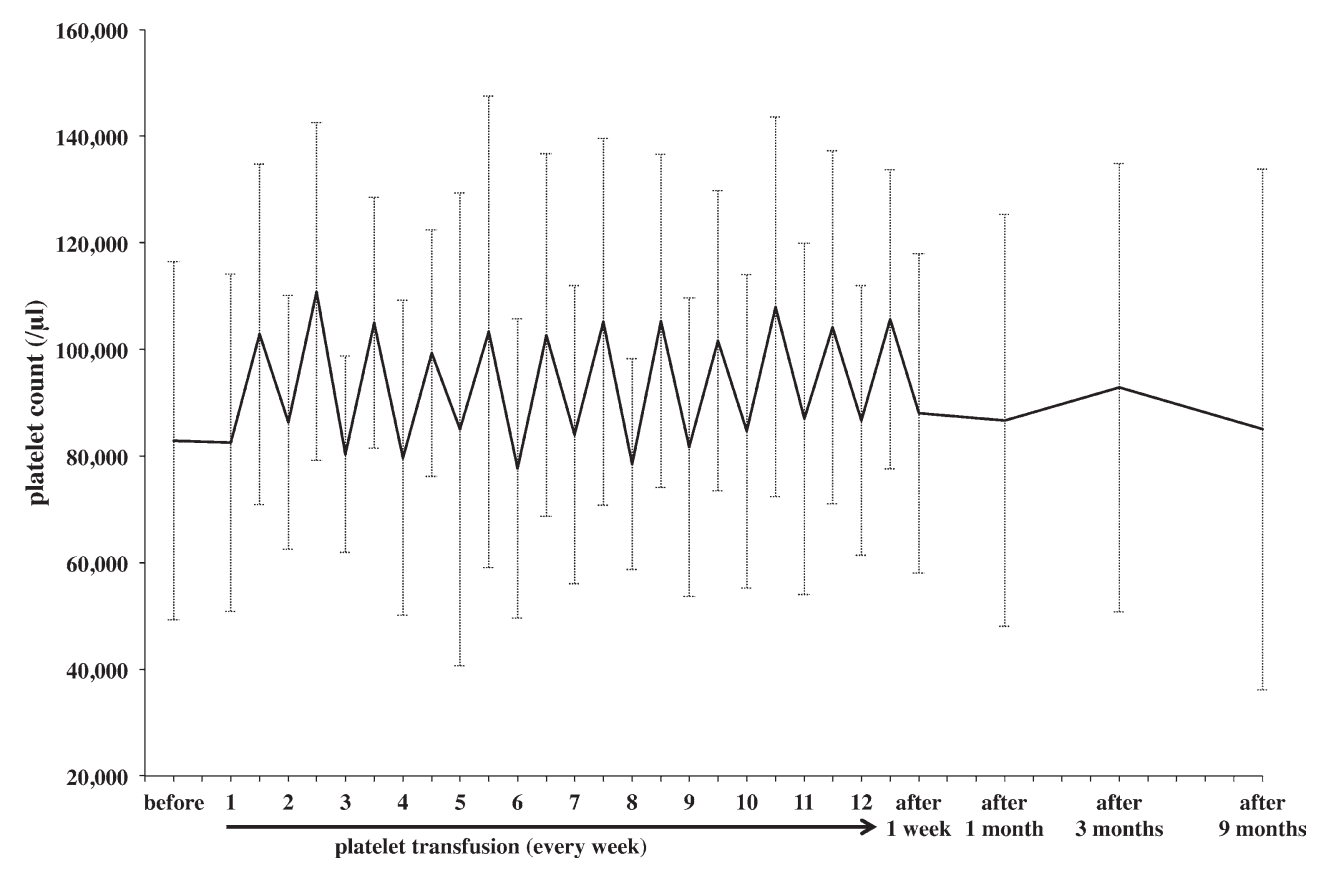

Fig. 3. Changes in platelet count.

The mean platelet count of the analyzed patients $(n=6)$ is presented. The platelet count increased by $20,000-30,000 / \mu 1$ at 1 hour after each platelet transfusion and then declined to almost the baseline level a week later. The platelet count did not increase significantly after the last transfusion, compared with the count before platelet transfusion. The data are expressed as the means \pm standard deviations.

patients $(n=6)$ are shown in Fig. 3. The platelet count increased by 20,000-30,000/ $\mu$ l at 1 hour after each platelet transfusion and decreased to almost the baseline level a week later. The platelet counts at 1 week, 1 month, 3 months, and 9 months after the last transfusion were 88,000 $\pm 30,000 / \mu \mathrm{l}(p=0.89), 86,700 \pm 38,600 / \mu \mathrm{l}(p=0.96)$, $92,800 \pm 42,100 / \mu \mathrm{l}(p=0.46)$, and 85,000 $\pm 48,800 / \mu \mathrm{l}$ $(p=1.00)$, respectively, none of which was significantly different from the value before platelet transfusion $(82,800$ $\pm 33,600 / \mu 1)$. The values of serum albumin at 1 month and 3 months after the last transfusion were $3.82 \pm 0.33 \mathrm{~g} / \mathrm{dl}$ $(p<0.05)$ and $3.80 \pm 0.25 \mathrm{~g} / \mathrm{dl}(p<0.05)$, respectively, which were significantly higher than the value before platelet transfusion $(3.43 \pm 0.40 \mathrm{~g} / \mathrm{dl})$ (Fig. 4A). The values of serum albumin at 1 week and 9 months after the last transfusion were $3.73 \pm 0.38 \mathrm{~g} / \mathrm{dl}(p=0.10)$ and $3.68 \pm 0.33 \mathrm{~g} / \mathrm{dl}$ 
(A) Serum albumin

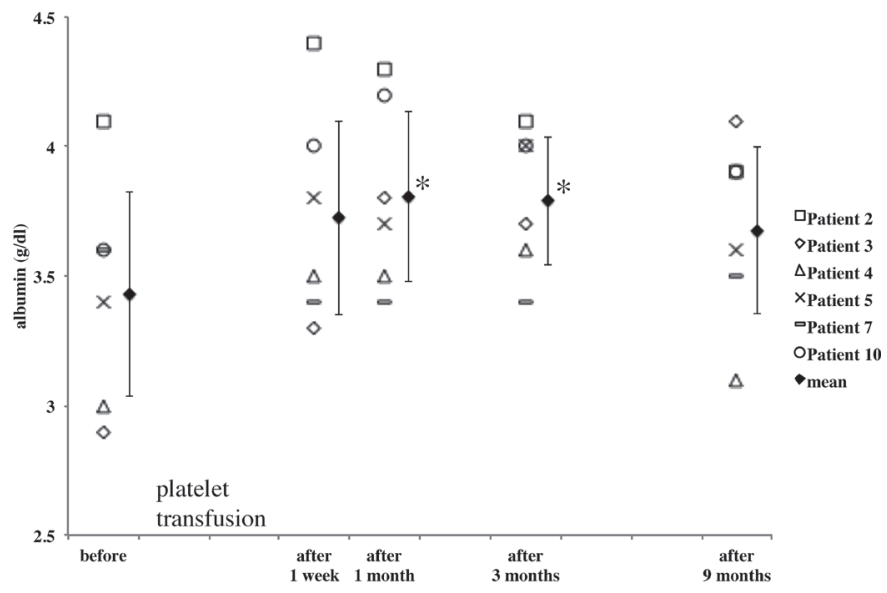

(B) Serum cholinesterase

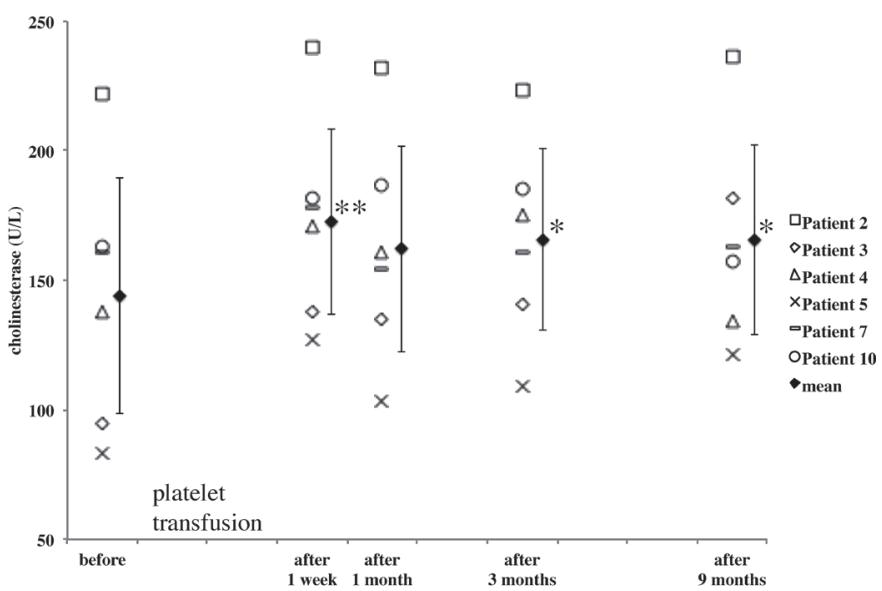

(C) Serum hyaluronic acid

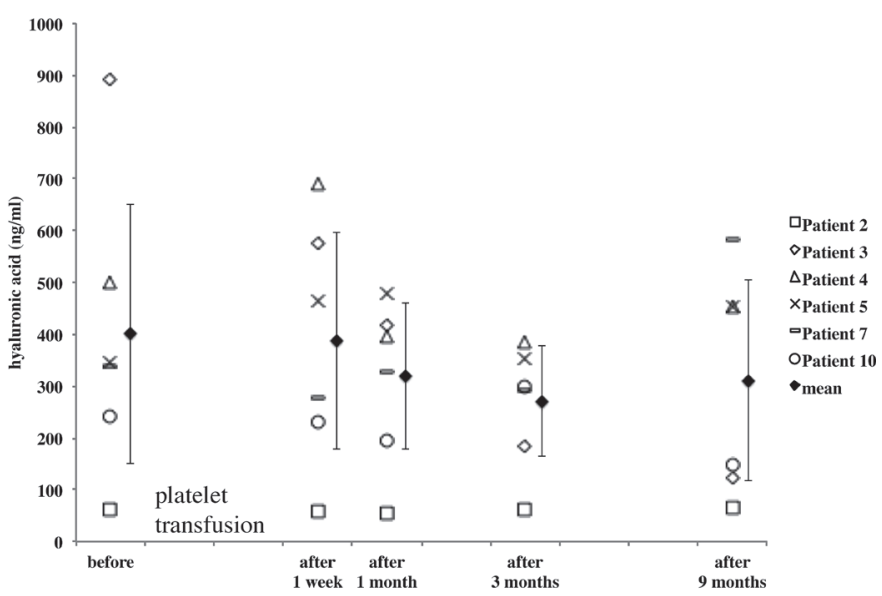

$* * p<0.01, * \mathrm{p}<0.05$ compare to the values before platelet transfusion

Fig. 4. Liver function improvements after platelet transfusion.

(A) Serum albumin, (B) serum cholinesterase, and (C) serum hyaluronic acid. (A) Serum albumin at 1 month $(p<0.05)$ and 3 months $(p<0.05)$ after the last transfusion was significantly higher than before platelet transfusion. (B) Serum cholinesterase at 1 week $(p<0.01), 3$ months $(p<0.05)$, and 9 months $(p<0.05)$ after the last transfusion was significantly higher than before platelet transfusion. (C) Serum hyaluronic acid after the last transfusion improved compared with before platelet transfusion, but not significantly. The data are expressed as the means \pm standard deviations. ${ }^{*} p<$ $0.05, * * p<0.01$ compared with the levels before platelet transfusion.

$(p=0.22)$, respectively, which showed a trend toward improvement but were not significantly different from the baseline. The values of serum cholinesterase at 1 week, 3 months, and 9 months after the last transfusion were 172.7 $\pm 36.4 \mathrm{IU} / 1(p<0.01), 165.7 \pm 35.6 \mathrm{IU} / 1(p<0.05)$, and $165.5 \pm 37.2 \mathrm{IU} / 1(p<0.05)$, respectively, which were significantly higher than the value before platelet transfusion (143.7 $\pm 50.8 \mathrm{IU} / \mathrm{l})$ (Fig. 4B). The value of serum cholines- 
terase at 1 month after the last transfusion was $162.0 \pm 40.4$ IU/1 $(p=0.11)$, which showed a trend toward improvement but was not significantly different from the baseline. The values of serum hyaluronic acid at 1 week, 1 month, 3 months, and 9 months after the last transfusion were 383.0 $\pm 215.5 \mathrm{ng} / \mathrm{ml}(p=1.00), 311.8 \pm 144.7 \mathrm{ng} / \mathrm{ml}(p=0.73)$, $263.0 \pm 109.4 \mathrm{ng} / \mathrm{ml}(p=0.36)$, and $304.0 \pm 198.7 \mathrm{ng} / \mathrm{ml}$ ( $p=0.67$ ), respectively, which were slight but not significant improvements compared to the value before platelet transfusion $(396.8 \pm 257.6 \mathrm{ng} / \mathrm{ml})$ (Fig. 4C). Neither serum type IV collagen nor P-III-P was significantly improved (data not shown). In the CT volumetry analysis, the liver volumes after platelet transfusion did not increase significantly compared with the volumes before platelet transfusion (data not shown).

\section{Discussion}

Platelet transfusion is an established therapy for thrombocytopenia, with well-known benefits and complications (Stroncek and Rebulla 2007). Platelets promote hepatocyte proliferation and have an anti-fibrotic effect in vitro (Matsuo et al. 2008; Kawasaki et al. 2010; Ikeda et al. 2011), and thrombocytosis promotes liver regeneration and reduces liver fibrosis in vivo (Murata et al. 2007; Murata et al. 2008b; Myronovych et al. 2008; Watanabe et al. 2009). We hypothesized that platelet increment therapy would improve liver function in humans. In this exploratory clinical trial, we observed that platelet transfusion improved some of the indicators of liver function in patients with CLD and cirrhosis, but some adverse events were associated with platelet transfusion, such as pruritus, hives, and PTR.

Serum albumin and cholinesterase improved significantly after platelet transfusion. Serum hyaluronic acid showed a trend toward improvement. When the liver is injured, platelets accumulate in the injured liver and actively translocate into Disse spaces (Endo and Nakamura 1992, 1993; Nakamura et al. 1998; Murata et al. 2007; Myronovych et al. 2008). The direct contact between platelets and hepatocytes or LSECs is necessary for hepatocyte proliferation (Matsuo et al. 2008; Kawasaki et al. 2010), and the direct contact between platelets and HSCs is essential for an anti-fibrotic effect (Ikeda et al. 2011). Taken together, the previous and current data suggest that transfused platelets delivered to the portal vein accumulate in the damaged liver due to CLD; directly contact hepatocytes, LSECs, and HSCs; and finally improve liver function and reduce liver fibrosis.

After an initial improvement up to 3 months, serum albumin at 9 months after the last transfusion gradually decreased to near the pre-platelet transfusion value. One potential reason why the beneficial effect did not persist in the long term is that the transfused platelets disappeared within 1 week; thus, the platelet count returned to the baseline value. If the patients with thrombocytopenia could maintain thrombocytosis for a long period, liver function would improve for an extended period. Splenectomy and partial splenic embolization induce thrombocytosis and improve liver function for more than 6 months in patients with CLD and cirrhosis (Murata et al. 1996; Murata et al. 2008a). However, patients with CLD and cirrhosis often have splenomegaly and thrombocytopenia and are at high risk for bleeding and complications in operations (Patel et al. 2003; Ohta et al. 2005). Additionally, partial splenic embolization has various complications, such as ascites, splenic or portal vein thrombosis, splenic abscess, and splenic necrosis. In addition to splenectomy and partial splenic embolization, administration of thrombopoietin is another method for inducing thrombocytosis. Orally active thrombopoietin receptor agonist increases platelet count in patients with thrombocytopenia due to hepatitis $\mathrm{C}$ virusrelated CLD and cirrhosis (McHutchison et al. 2007). Thrombocytosis by administering thrombopoietin improves liver regeneration and reduces liver fibrosis in vivo (Murata et al. 2007; Murata et al. 2008b; Myronovych et al. 2008; Watanabe et al. 2009). Therefore, thrombocytosis by administration of thrombopoietin could help sustain the improvement of liver function in patients with CLD and cirrhosis.

When we began this trial, there were no data regarding the concentration of platelets that could improve liver function in patients with CLD and cirrhosis. We already found that human platelet transfusion to mice promoted liver regeneration after hepatectomy (Takahashi et al. 2012). In this study, the number of transfused human platelets was between 10 and $20 \%$ of the total number of platelets in mice. Thrombocytosis by splenectomy improved liver function in patients with CLD and cirrhosis after 3 months (Murata et al. 2008a). These findings helped us determine the concentration and duration of platelet transfusion in this trial. In patient 6 , who discontinued platelet transfusion because of PTR after the third transfusion, serum cholinesterase at 1 month after the last transfusion increased compared with the value before platelet transfusion (data not shown). Using a smaller amount of platelets could improve liver function. However, in patient 9, who received 12 platelet transfusions, serum albumin and cholinesterase did not increase (date not shown). One reason why the liver function of this patient did not improve might be that the transfused platelets would not pass through the portal vein because he had a major splenorenal shunt. For those patients who have major shunts and hepatofugal collaterals, such as esophagogastric varices and splenorenal shunts, platelet transfusion might be less effective.

We evaluated liver fibrosis in patients by means of blood markers. Liver fibrosis should be assessed from not only a hematological perspective but also based on histopathological and morphological findings. Although liver biopsy is a standard histopathological method for evaluating liver fibrosis, this invasive procedure has a great risk of bleeding in patients with thrombocytopenia (Seeff et al. 2010). For this reason, we did not perform liver biopsy in 
this trial. Transient elastography is a non-invasive method for evaluating hepatic fibrosis by measuring liver stiffness, and it can be easily performed in the outpatient clinic with immediate results and good reproducibility (Castera et al. 2008). Liver fibrosis is thus better assessed by transient elastography.

We did not observe any fatal adverse events, such as thrombosis, anaphylactic shock or transfusion-related acute lung injury (TRALI), in this trial. However, 2 of 10 patients exhibited PTR by repeated platelet transfusion; both of these patients were females with histories of pregnancy. PTR occurred at approximately the same rate as in previous reports, from $13 \%$ to $34 \%$ (Klingemann et al. 1987; Legler et al. 1997; The Trial to Reduce Alloimmunization to Platelets Study Group. 1997). A patient with a history of pregnancy has a greater risk of PTR (The Trial to Reduce Alloimmunization to Platelets Study Group. 1997) and should be closely monitored when they repeatedly receive platelet transfusions. In addition, we observed non-hemolytic transfusion reactions (NHTRs), such as pruritus and hives. NHTRs are a relatively common complication of allogeneic transfusion, and the incidence of NHTRs in platelet concentrate is greater than in red blood cells or fresh frozen plasma (Kurata et al. 2007). In this trial, NHTRs occurred in $4.3 \%$ of platelet transfusions, similar to the $4.2 \%$ reported in a study in Japan (Kurata et al. 2007). Repeated platelet transfusions in patients with CLD and cirrhosis should not increase the incidence of NHTRs.

\section{Conclusion}

Platelet transfusion improved some of the indicators of liver function in patients with CLD and cirrhosis, and platelet increment therapy could be a novel strategy for the treatment of CLD and cirrhosis. Adverse events related to platelet transfusion were observed at approximately the same rate as in previous reports.

\section{Acknowledgments}

The authors thank the Tsukuba Critical Path Research and Education Integrated Leading Center, Graduate School of Comprehensive Human Sciences, University of Tsukuba, for coordinating this trial.

\section{Conflict of Interest}

The authors declare no conflict of interest.

\section{References}

Adinolfi, L.E., Giordano, M.G., Andreana, A., Tripodi, M.F., Utili, R., Cesaro, G., Ragone, E., Durante Mangoni, E. \& Ruggiero, G. (2001) Hepatic fibrosis plays a central role in the pathogenesis of thrombocytopenia in patients with chronic viral hepatitis. Br. J. Haematol., 113, 590-595.

Afdhal, N., McHutchison, J., Brown, R., Jacobson, I., Manns, M., Poordad, F., Weksler, B. \& Esteban, R. (2008) Thrombocytopenia associated with chronic liver disease. J. Hepatol., 48, 1000-1007.

Anitua, E., Andia, I., Ardanza, B., Nurden, P. \& Nurden, A.T.
(2004) Autologous platelets as a source of proteins for healing and tissue regeneration. Thromb. Haemost., 91, 4-15.

Assoian, R.K., Komoriya, A., Meyers, C.A., Miller, D.M. \& Sporn, M.B. (1983) Transforming growth factor-beta in human platelets. Identification of a major storage site, purification, and characterization. J. Biol. Chem., 258, 7155-7160.

Bashour, F.N., Teran, J.C. \& Mullen, K.D. (2000) Prevalence of peripheral blood cytopenias (hypersplenism) in patients with nonalcoholic chronic liver disease. Am. J. Gastroenterol., 95, 2936-2939.

Castera, L., Forns, X. \& Alberti, A. (2008) Non-invasive evaluation of liver fibrosis using transient elastography. J. Hepatol., 48, 835-847.

D’Amico, G., Garcia-Tsao, G. \& Pagliaro, L. (2006) Natural history and prognostic indicators of survival in cirrhosis: a systematic review of 118 studies. J. Hepatol., 44, 217-231.

Daly, P.A., Schiffer, C.A., Aisner, J. \& Wiernik, P.H. (1980) Platelet transfusion therapy. One-hour posttransfusion increments are valuable in predicting the need for HLA-matched preparations. JAMA, 243, 435-438.

Diegelmann, R.F. \& Evans, M.C. (2004) Wound healing: an overview of acute, fibrotic and delayed healing. Front. Biosci., 9, 283-289.

Endo, Y. \& Nakamura, M. (1992) The effect of lipopolysaccharide, interleukin-1 and tumour necrosis factor on the hepatic accumulation of 5-hydroxytryptamine and platelets in the mouse. Br. J. Pharmacol., 105, 613-619.

Endo, Y. \& Nakamura, M. (1993) Active translocation of platelets into sinusoidal and Disse spaces in the liver in response to lipopolysaccharides, interleukin-1 and tumor necrosis factor. Gen. Pharmacol., 24, 1039-1053.

Friedman, S.L. (2008) Mechanisms of hepatic fibrogenesis. Gastroenterology, 134, 1655-1669.

Giannini, E., Borro, P., Botta, F., Fumagalli, A., Malfatti, F., Podesta, E., Romagnoli, P., Testa, E., Chiarbonello, B., Polegato, S., Mamone, M. \& Testa, R. (2002) Serum thrombopoietin levels are linked to liver function in untreated patients with hepatitis $\mathrm{C}$ virus-related chronic hepatitis. $J$. Hepatol., 37, 572-577.

Heidelbaugh, J.J. \& Sherbondy, M. (2006) Cirrhosis and chronic liver failure: part II. Complications and treatment. Am. Fam. Physician, 74, 767-776.

Ikeda, N., Murata, S., Maruyama, T., Tamura, T., Nozaki, R., Kawasaki, T., Fukunaga, K., Oda, T., Sasaki, R., Homma, M. \& Ohkohchi, N. (2011) Platelet-derived adenosine 5'-triphosphate suppresses activation of human hepatic stellate cell: in vitro study. Hepatol. Res., 42, 91-102.

Kawasaki, T., Murata, S., Takahashi, K., Nozaki, R., Ohshiro, Y., Ikeda, N., Pak, S., Myronovych, A., Hisakura, K., Fukunaga, K., Oda, T., Sasaki, R. \& Ohkohchi, N. (2010) Activation of human liver sinusoidal endothelial cell by human platelets induces hepatocyte proliferation. J. Hepatol., 53, 648-654.

Klingemann, H.G., Self, S., Banaji, M., Deeg, H.J., Doney, K., Slichter, S.J., Thomas, E.D. \& Storb, R. (1987) Refractoriness to random donor platelet transfusions in patients with aplastic anaemia: a multivariate analysis of data from 264 cases. $J$. Haematol., 66, 115-121.

Kurata, Y., Shimizu, M., Okazaki, H. \& Takemoto, S. (2007) Incidence of Nonhemolytic Transfusion Reaction due to Immnological Reactions. Japanese Journal of Transfusion and Cell Therapy, 53, 43-46.

Legler, T.J., Fischer, I., Dittmann, J., Simson, G., Lynen, R., Humpe, A., Riggert, J., Schleyer, E., Kern, W., Hiddemann, W. \& Kohler, M. (1997) Frequency and causes of refractoriness in multiply transfused patients. Ann. Hematol., 74, 185-189.

Malik, R., Selden, C. \& Hodgson, H. (2002) The role of nonparenchymal cells in liver growth. Semin. Cell. Dev. Biol., 13, 425-431.

Matsuo, R., Ohkohchi, N., Murata, S., Ikeda, O., Nakano, Y., 
Watanabe, M., Hisakura, K., Myronovych, A., Kubota, T., Narimatsu, H. \& Ozaki, M. (2008) Platelets strongly induce hepatocyte proliferation with IGF-1 and HGF in vitro. J. Surg. Res., 145, 279-286.

McHutchison, J.G., Dusheiko, G., Shiffman, M.L., RodriguezTorres, M., Sigal, S., Bourliere, M., Berg, T., Gordon, S.C., Campbell, F.M., Theodore, D., Blackman, N., Jenkins, J. \& Afdhal, N.H. (2007) Eltrombopag for thrombocytopenia in patients with cirrhosis associated with hepatitis C. N. Engl. J. Med., 357, 2227-2236.

Merion, R.M., Schaubel, D.E., Dykstra, D.M., Freeman, R.B., Port, F.K. \& Wolfe, R.A. (2005) The survival benefit of liver transplantation. Am. J. Transplant., 5, 307-313.

Miyazono, K. \& Takaku, F. (1989) Platelet-derived growth factors. Blood. Rev., 3, 269-276.

Mueller, A.R., Platz, K.P. \& Kremer, B. (2004) Early postoperative complications following liver transplantation. Best. Pract. Res. Clin. Gastroenterol., 18, 881-900.

Murata, K., Ito, K., Yoneda, K., Shiraki, K., Sakurai, H. \& Ito, M. (2008a) Splenectomy improves liver function in patients with liver cirrhosis. Hepatogastroenterology, 55, 1407-1411.

Murata, K., Shiraki, K., Takase, K., Nakano, T. \& Tameda, Y. (1996) Long term follow-up for patients with liver cirrhosis after partial splenic embolization. Hepatogastroenterology, 43, 1212-1217.

Murata, S., Hashimoto, I., Nakano, Y., Myronovych, A., Watanabe, M. \& Ohkohchi, N. (2008b) Single administration of thrombopoietin prevents progression of liver fibrosis and promotes liver regeneration after partial hepatectomy in cirrhotic rats. Ann. Surg., 248, 821-828.

Murata, S., Ohkohchi, N., Matsuo, R., Ikeda, O., Myronovych, A. \& Hoshi, R. (2007) Platelets promote liver regeneration in early period after hepatectomy in mice. World J. Surg., 31, 808-816.

Myronovych, A., Murata, S., Chiba, M., Matsuo, R., Ikeda, O., Watanabe, M., Hisakura, K., Nakano, Y., Kohno, K., Kawasaki, T., Hashimoto, I., Shibasaki, Y., Yasue, H. \& Ohkohchi, N. (2008) Role of platelets on liver regeneration after 90\% hepatectomy in mice. J. Hepatol., 49, 363-372.

Nakamura, M., Shibazaki, M., Nitta, Y. \& Endo, Y. (1998) Translocation of platelets into Disse spaces and their entry into hepatocytes in response to lipopolysaccharides, interleukin-1 and tumour necrosis factor: the role of Kupffer cells. $J$. Hepatol., 28, 991-999.

Nakamura, T., Teramoto, H. \& Ichihara, A. (1986) Purification and characterization of a growth factor from rat platelets for mature parenchymal hepatocytes in primary cultures. Proc. Natl. Acad. Sci. USA, 83, 6489-6493.

Neuberger, J. \& James, O. (1999) Guidelines for selection of patients for liver transplantation in the era of donor-organ shortage. Lancet, 354, 1636-1639.

Ohta, M., Nishizaki, T., Matsumoto, T., Shimabukuro, R., Sasaki, A., Shibata, K., Matsusaka, T. \& Kitano, S. (2005) Analysis of risk factors for massive intraoperative bleeding during laparoscopic splenectomy. J. Hepatobiliary Pancreat. Surg., 12, 433-437.

Oka, Y. \& Orth, D.N. (1983) Human plasma epidermal growth factor/beta-urogastrone is associated with blood platelets. $J$. Clin. Invest., 72, 249-259.

Patel, A.G., Parker, J.E., Wallwork, B., Kau, K.B., Donaldson, N., Rhodes, M.R., O'Rourke, N., Nathanson, L. \& Fielding, G. (2003) Massive splenomegaly is associated with significant morbidity after laparoscopic splenectomy. Ann. Surg., 238, 235-240.

Porrett, P.M., Hsu, J. \& Shaked, A. (2009) Late surgical complications following liver transplantation. Liver Transpl., 15 Suppl 2, S12-18.

Seeff, L.B., Everson, G.T., Morgan, T.R., Curto, T.M., Lee, W.M., Ghany, M.G., Shiffman, M.L., Fontana, R.J., Di Bisceglie, A.M., Bonkovsky, H.L. \& Dienstag, J.L. (2010) Complication rate of percutaneous liver biopsies among persons with advanced chronic liver disease in the HALT-C trial. Clin. Gastroenterol. Hepatol., 8, 877-883.

Stroncek, D.F. \& Rebulla, P. (2007) Platelet transfusions. Lancet, 370, 427-438.

Takahashi, K., Kozuma, Y., Suzuki, H., Tamura, T., Maruyama, T., Fukunaga, K., Murata, S. \& Ohkohchi, N. (2012) Human platelets promote liver regeneration with Kupffer cells in SCID mice. J. Surg. Res., Epub ahead of print.

Taylor, M.C., Greig, P.D., Detsky, A.S., McLeod, R.S., Abdoh, A. \& Krahn, M.D. (2002) Factors associated with the high cost of liver transplantation in adults. Can. J. Surg., 45, 425-434.

The Trial to Reduce Alloimmunization to Platelets Study Group (1997) Leukocyte reduction and ultraviolet B irradiation of platelets to prevent alloimmunization and refractoriness to platelet transfusions. N. Engl. J. Med., 337, 1861-1869.

Watanabe, M., Murata, S., Hashimoto, I., Nakano, Y., Ikeda, O., Aoyagi, Y., Matsuo, R., Fukunaga, K., Yasue, H. \& Ohkohchi, N. (2009) Platelets contribute to the reduction of liver fibrosis in mice. J. Gastroenterol. Hepatol., 24, 78-89. 